\title{
Full 3D displacement field measurement by Optical Scanning Tomography and PIV Tomography
}

\author{
A. Germaneau ${ }^{1}$, P. Doumalin ${ }^{1}$, J.-C. Dupré ${ }^{1}$, L. Thomas ${ }^{1}$, V. Grulier ${ }^{1}$, P. Braud ${ }^{1}$, B. Tremblais ${ }^{2}$, \\ L. David ${ }^{1}$ \\ ${ }^{1}$ Institut Pprime, UPR 3346 CNRS - Université de Poitiers - ENSMA \\ S.P.2M.I., Bd Marie et Pierre Curie, Téléport 2, 86960 Futuroscope Chasseneuil, France \\ ${ }^{2}$ Laboratoire XLIM-Signal Image Communication, Université de Poitiers, CNRS \\ S.P.2M.I., Bd Marie et Pierre Curie, Téléport 2, 86960 Futuroscope Chasseneuil, France
}

\begin{abstract}
This work is about the development of new techniques for the measurement of the three components of the velocity inside a volume. A comparison of two techniques, optical scanning tomography and algebraic reconstruction tomography method, is performed with 3D displacement tests of transparent solid blocks.
\end{abstract}

\section{3D measurement fields}

This work is about the development of new techniques to study 3D mechanical problems involved inside a volume, in particular in the framework of fluid mechanics. For that, Tomographic Particle Image Velocimetry (Tomo-PIV) has been developed to measure the three components of the velocity in a whole volume [1]. This technique consists in recording some projections from different orientations and in using an algebraic reconstruction method to generate a volume image of particles. Optical scanning tomography is another method to generate volumes images and is also an alternative to record the volume particles. In this paper, reconstructions of particle volumes by MART algebraic tomography [2-4] and by optical scanning tomography [5,6] are compared on rigid body motion tests. For that, resin blocks have been manufactured with different particle diameters and particle rates and are moved in a tank filled with a fluid resin to suppress the effect of refraction index. For the same position, blocks are recorded by four cameras for the multi sensor tomography and by one camera perpendicular to the laser beam for multi-plane tomography. Particle volumes reconstructed by both techniques are compared. Accuracy of the reconstruction, drawbacks and limitations are presented and discussed. For the different positions of the blocks, Digital Volume Correlation (DVC), the 3D extension of Digital Image Correlation (DIC), is used to measure displacement uncertainty and evaluate the influence of each tomography method.

\section{Multi-sensor tomography}

Currently the main techniques used in tomography for the volumic reconstruction in fluid mechanics fluids are ART algebraic reconstruction (Algebraic Reconstruction Technic) and MART (Multiplicative ART). Indeed, these methods are well adapted to reconstruct a volume starting from a very limited number of views (2 to 6 ). The problem of reconstruction can be written like a linear

This is an Open Access article distributed under the terms of the Creative Commons Attribution-Noncommercial License 3.0, which permits unrestricted use, distribution, and reproduction in any noncommercial medium, provided the original work is properly cited. 
system of equations since the contribution of the intensities of the voxels of a volume to the intensities of the pixels of the views and is given by $W . X=Y$ where $Y$ and $X$ are respectively the vector intensity of the pixels and the vector intensity of the voxels. $\mathrm{W}$ is called matrix of weight or of influency. The row $p$ of $W$ represents a ray of projection on the pixel $p$ interacting with voxels $v$ of volume in the vicinity of this ray:

$$
\sum_{v} w_{p v} X_{v}=Y_{p}
$$

where $Y_{p}$ is the intensity of the pixel $p, X_{v}$ is the intensity of the voxel $v, p=1, \ldots, N_{p}$ with $N_{p}$ the number of pixels and $v=1, \ldots, N_{v}$ with $N_{v}$ the number of voxels. The coefficient $w_{p v}$ is a weight which characterizes the influence of the voxel $v$ on the pixel $p$. The coefficients $w_{i j}$ are computed to leave a preliminary stage which is the calibration of the cameras. The calibration gives the lines of sight or rays of projection associated with each pixel of the cameras. We calculate the coefficients in the following way: if the line of sight of pixel p intercepts the voxel $v$ then the matrix of weight takes a unit value with cross of row $p$ and column $v$. Conversely if it does not intercept the voxel then the coefficient $w_{p v}$ remains null (Figure 1).

(a)

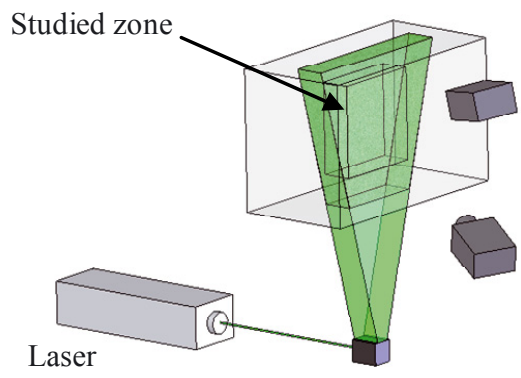

(b)

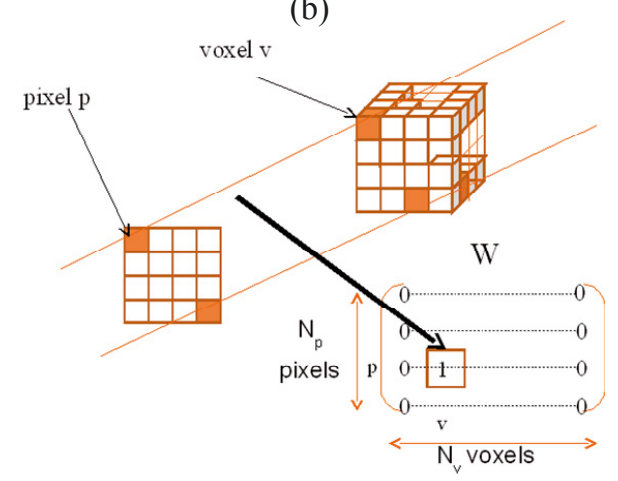

Figure 1. (a) Principle of multi-sensor tomography; (b) Schematization of the interaction matrix

The algebraic methods consist in getting the value of $X$ which minimizes the functional $J(X)=\| Y$ $\left.W X\right|^{2}$. The system of equations can be divided into several subsystems or blocks of equations. Each block then will contain one or more rays of projection and thus one or more linear equations of the system. In a general way the ART algorithms are iterative algorithms which correspond to the mathematical method of Kaczmarz [7] for the resolution of the linear system of equations. The MART algorithm consists on a multiplicative actualization of the intensity of the voxels based on the ratio of the measured intensity of the pixel and the projection of the intensities of the voxels obtained at the preceding iteration:

$$
X_{v}^{(k+1)}=X_{v}^{(k)}\left(\frac{Y_{p}}{\sum_{j} w_{p j} X_{j}^{(k)}}\right)^{\mu w_{p v}}
$$

where $\mu$ is the scalar relaxation coefficient such as $\mu \leq 1$. An iteration MART is complete when all the pixels $\mathrm{p}$ were considered. The coefficient of relaxation $\mu$ can be optimized for a faster convergence. For this application, four $1600 \times 1200$ pixels JAI CCD cameras are used with Scheimpflug arrangements [8]. Resin block of particles is placed in a tank filled with fluid of index to remove the effects of refraction index for passages air, tank, fluid, block (Figure 2). Two CCD cameras are placed with an angle of $+/-45^{\circ}$ compared to $Z$ in a plan $X Z$ and two others are placed with an angle of $+/-20^{\circ}$ compared to $Z$ in a plan $Y Z$. The tank has two faces with $45^{\circ}$ and a prism, having an angle of $140^{\circ}$ is stuck against one of its faces, which allow a recording of the block without distortion or astigmatism. The zone of recording is highlighted by a continuous laser. The 
pinhole model is used for each camera and the common area for the reconstruction is approximately a volume of $90 \mathrm{~mm} \times 80 \mathrm{~mm} \times 15 \mathrm{~mm}$.

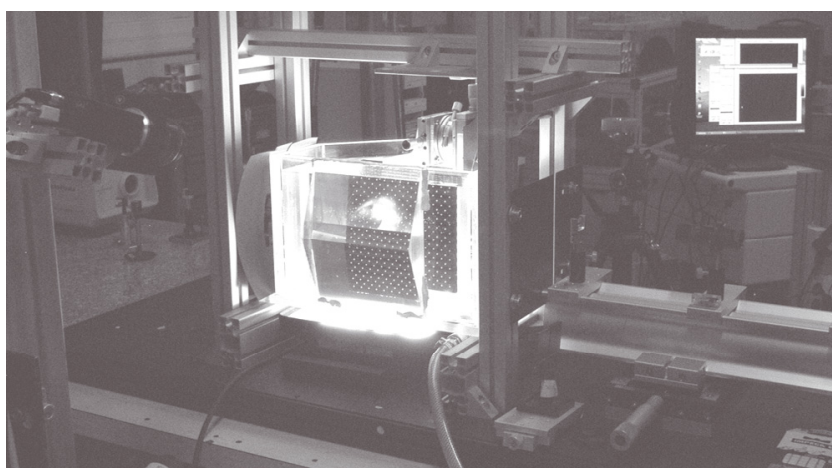

Figure 2. Experimental Setup of the block measurement

\section{Optical scanning tomography}

Optical scanning tomography is based on the phenomenon of scattered light created by randomly distributed particles, added in the specimen during its elaboration. At each imposed displacement, a $3 \mathrm{D}$ image is obtained by scanning the specimen with a plane laser beam in the $\mathrm{z}$ direction and with a motorized translation stage controlled by an integrated linear-scale encoder (Figure 3). Scanning is performed by moving either the laser or resin blocks. Two solutions are employed to scan at each position of the beam, we record an $x-y$ 2D image of the illuminated section where a random pattern due to scatter particles appears. The volume is constituted by the succession of these 2D images (Figure 4). The optical device consists of a laser source, a convergent lens and a cylinder lens which will form a laser plan (Figure 3). To obtain cubic voxels in the volume image, the resolution of CCD camera ( $x-y$ plane there) must be equal to the step imposed between two successive scans in direction $Z$. To have a fill factor of voxels equal to $100 \%$, the thickness of the plane laser beam must be equal to the step between two successive slices [6].

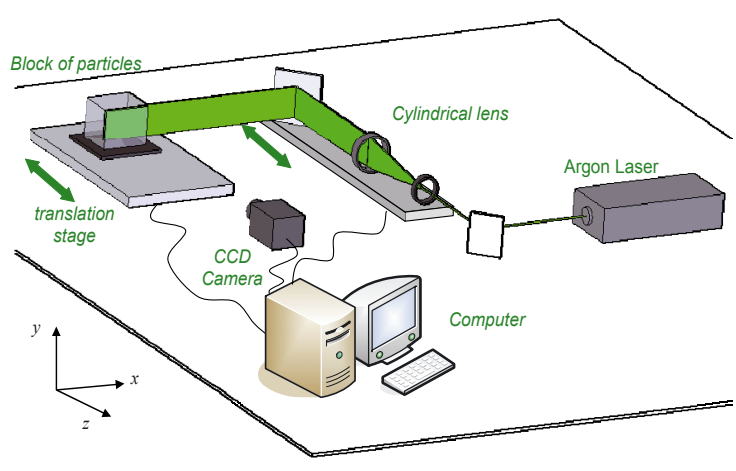

Figure 3. Experimental Setup for scanning

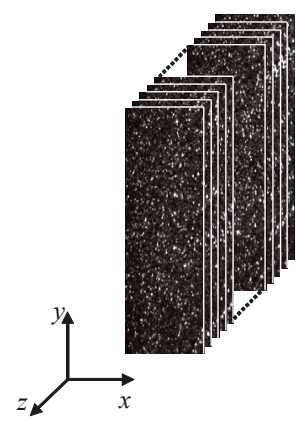

Figure 4. Volume Image Reconstruction

The plane laser beam thickness is about $60 \mu \mathrm{m}$ and volumes are recorded by a 5 th camera of $1640 \times 1420$ pixels located along the $Z$ axis with a focal lens equal to $50 \mathrm{~mm}$, a F-number of 5.6 and a pixel size of $5 \mu \mathrm{m}$. 


\section{Digital Volume Correlation}

Digital volume correlation is the $3 \mathrm{D}$ extension of the correlation of $2 \mathrm{D}$ images which is usually used for the measurement of displacements in a plane. This technique makes it possible to measure the displacement field between two different times (or two locations here) in the middle of the studied block. The displacement of each point of this virtual grid is calculated by intercorrelation of the grey levels of the neighborhood $D$ surrounding the considered point in both states. $D$ is composed of several voxels and corresponds to a subset of the volume. By noting $X$ and $x$ the $3 \mathrm{D}$ coordinates (in voxels) of a same point in the reference state and the deformed state, both configurations are linked by a 3D material transformation $\phi: x=\phi(X)$. For a subset $D$ centered at the point $X_{0}$ in the reference state, $\phi$ is approximated by its expansion at the first order corresponding to a rigid body motion combined with a homogeneous deformation. The best parameters characterizing the approximation are those which minimize a correlation coefficient $C$ which measures the degree of similarity of grey level distributions in $D$ and its transformed one by $\phi$. We choose a formulation of $C$ insensitive to small contrast and brightness fluctuations which can appear in images: a normalized cross correlation formulation based on grey level gaps in respect to the average on the subset:

$$
C=1-\frac{\sum_{\underline{X} \in D}\left(f(\underline{X})-\bar{f}_{D}\right) \cdot\left(g(\phi(\underline{X}))-\bar{g}_{D}\right)}{\sqrt{\sum_{\underline{X} \in D}\left(f(\underline{X})-\bar{f}_{D}\right)^{2}} \cdot \sqrt{\sum_{\underline{X} \in D}\left(g(\phi(\underline{X}))-\bar{g}_{D}\right)^{2}}}
$$

where $X$ refers to voxels in $D, f$ and $g$ are respectively the grey levels in the initial and translated images, $\bar{f}_{D}$ and $\bar{g}_{D}$ are their averages over $D$ and $\phi(D)$. A trilinear interpolation of the grey levels in the translated image is used in order to calculate the grey level variations between two adjacent voxels. In this way, it is possible to achieve the position of the subset $D$ in fractions of voxels (subvoxel precision). This solution is researched with an automatic first gradient minimization procedure from an estimation in entire voxels which is obtained by a direct systematic calculus

\section{Results}

\subsection{MART Reconstruction}

The MART reconstruction of the volume is performed with 5 steps of iteration and the relaxation parameter is fixed to one. The intensity is calculated over 2002 by 1441 by 167 voxels to have the same voxel size than with scanning tomography. When calculating the interactions between the pixels and the voxels, each pixel is divided in 100 subpixels to improve the precision of the reconstruction. Slices of the reconstructed volume in three directions are given on Figure 5. Even if we see clearly the particles on the three views, some depth effects appear on the $y-z$ and the $x-z$ views, corresponding to the lines of sight pixel-voxel. The result of the reconstruction without the use of subpixels is given on the $\mathrm{x}-\mathrm{y}$ slice of the Figure 5-d. By comparing this result with the one of the Figure 5-a, the beneficial contribution of the pixels oversampling is shown.

The number of subpixels is obviously important on the time of the reconstruction. On a computer with a good capacity of calculation, the reconstruction of a 2002 by 1441 by 167 voxels volume with five iterations takes 24 hours with 100 subpixels versus only less one hour if the pixels are not oversampled. Moreover the computation of a volume requires at less 2 Gigabytes of RAM.

In order to study the effects on the reconstruction of the parameters as the number of the iteration steps and the number of the subpixels, a particles volume is simulated and projected according to three views to make images. These images are the input data for the MART volume reconstruction. An error indicator is given by the RMS value of the difference between the original volume and the reconstructed one. Figures 6 and 7 show the variations of the error over respectively the number of iterations of the reconstruction algorithm and the number of subpixels. Figure 6 shows that a 
minimum number of iterations is necessary to the MART algorithm to converge. The best result is obtained around after twenty iterations (but ten iterations can be considered well converged). Over the curve shows that to exaggerate the numbers of iterations can make the reconstruction less efficient due to the divergence of the algorithm. The Figure 7 confirms quantitatively that we saw on the experimental results: the oversampling of the pixels is a good way to improve the reconstruction.

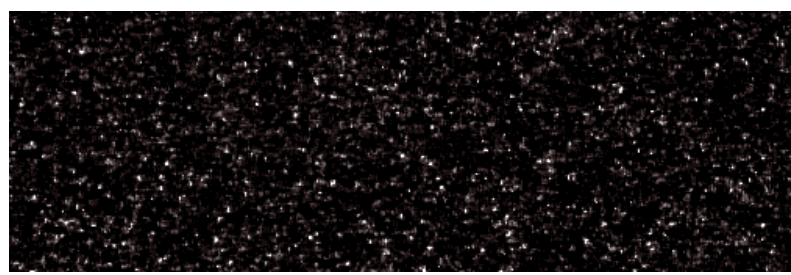

(a). $x-y$ slice.

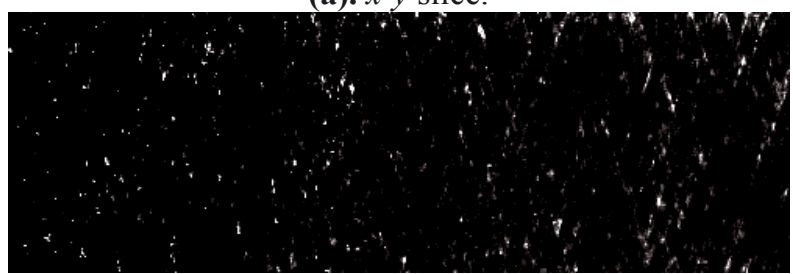

(b). $y$ - $z$ slice.

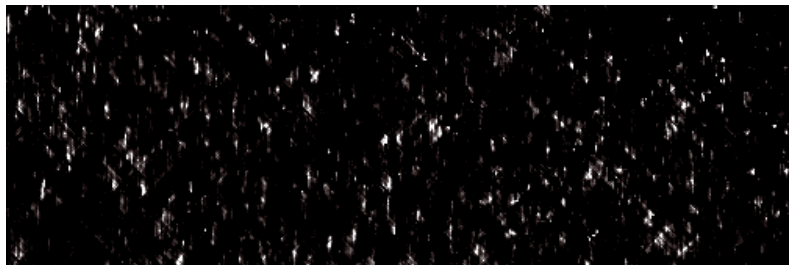

(c). $x-z$ slice.

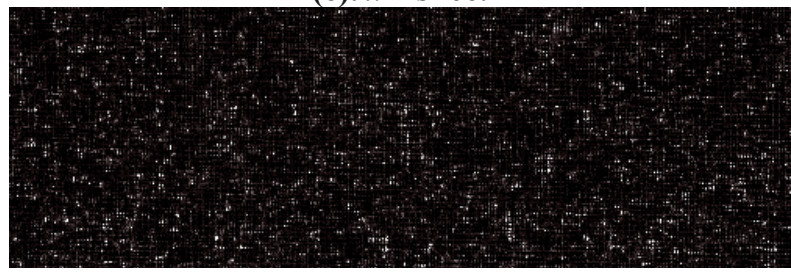

(d). $x-y$ slice. No pixels oversampling.

Figure 5. Slices of the reconstructed volume over three views.

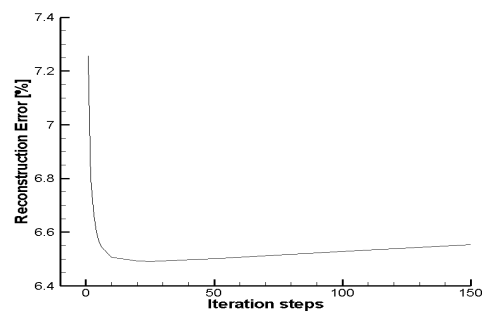

Figure 6. Reconstruction error over the number of iteration steps. 


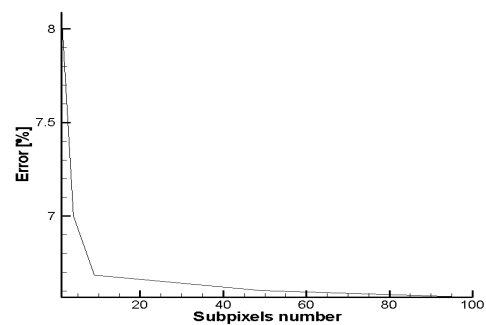

Figure 7. Reconstruction error over the number of subpixels number.

\subsection{Optical scanning tomography}

Two types of sweeping (fixed laser and moving block or fixed block and moving laser) are compared. Apparent dimensions of the objects depend on the focal lens of the camera, the distance between block and camera, the optical index of material, and on the depth corresponding to the optical path of scattered beam in the block. Figure 8 presents sections of volumes and shows that both techniques give rather similar images.

(a)

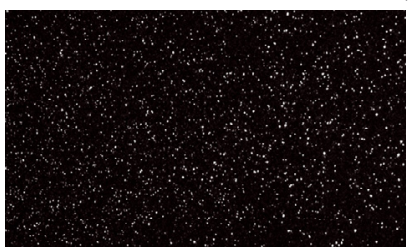

$x-y$

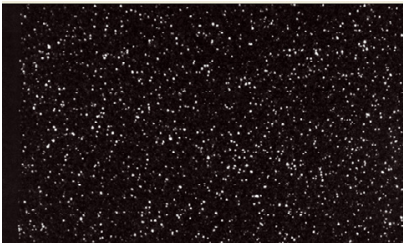

$x-y$

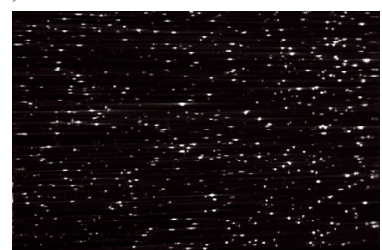

$z-y$

(b)

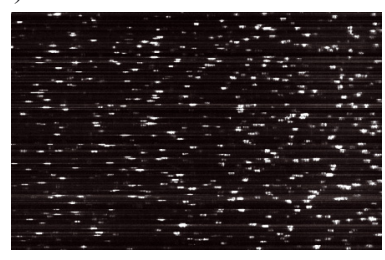

$z-y$

Figure 8. Slices within volume images recorded by optical scanning tomography with fixed laser (a) and moving laser (b)

In a quantitative way, Figure 9 shows that the first solution (moving block) gives less discrepancy according to the depth. This solution is currently employed in solid mechanics for static studies on structures [5]. For the flow study, only a scanning of the laser is possible. In the present case, the depth of volumes is of approximately $10 \mathrm{~mm}$ and apparent dimension variations remain weak (lower than $1 \%$ ) and can be neglected (or could be corrected by a preliminary calibration). 


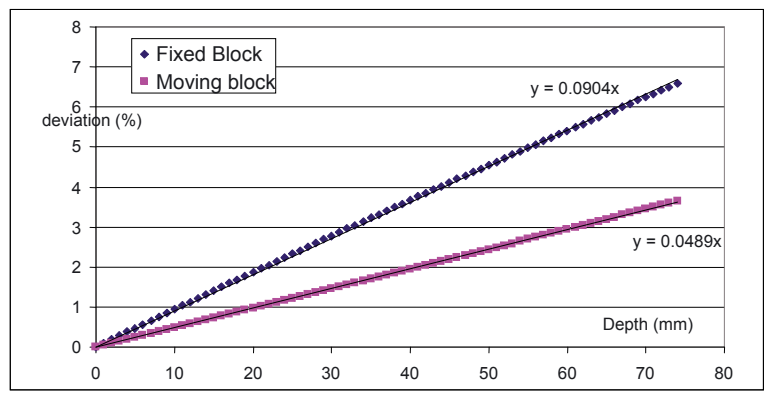

Figure 9. Apparent dimension variations in the $x-y$ plane according to scanning process

\subsection{Displacement results}

To evaluate measurement uncertainty and to compare both methods, displacement tests are performed on resin blocks containing polyamide powder. For each value of the imposed translation along y and $\mathrm{z}$ directions, volume images are recorded on one hand from multi-sensor tomography (MST) and on the other hand from optical scanning tomography (OST). Then, Digital Volume Calibration is employed to measure displacement field on a $3 \mathrm{D}$ grid within the volume. To evaluate systematic error, mean gaps between measured and imposed displacements are calculated and standard deviation values of these differences give measurement uncertainty (Figures 10 and 11).

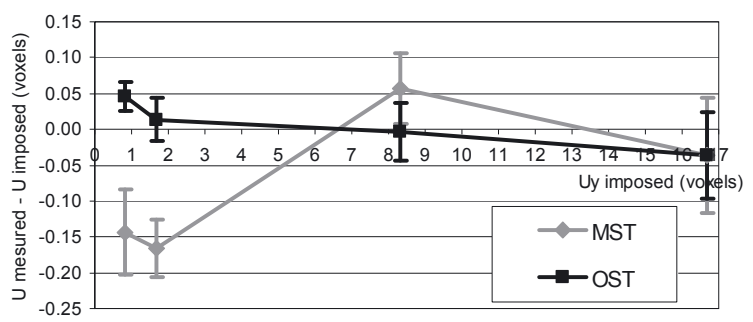

Figure 10. $y$-displacement measurement error by DVC for both methods according to y imposed displacement

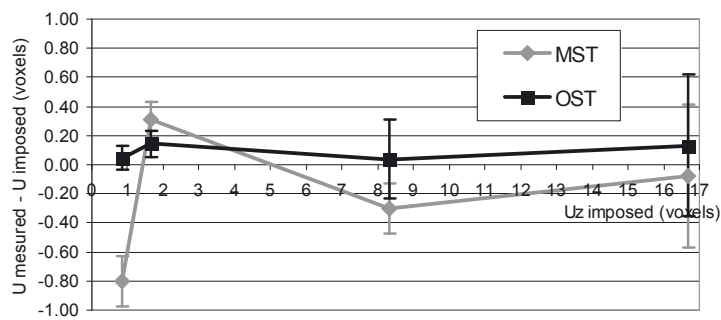

Figure 11. z-displacement measurement error by DVC for both methods according to z imposed displacement

These tests show that results are better by OST for small imposed displacements. It seems to be related directly to the voxel sampling. For larger displacement values, both methods give similar errors. 


\section{Conclusion}

A comparison of two techniques, optical scanning tomography and multi-sensor tomography method, is performed with 3D displacement tests of transparent solid blocks. Volume reconstruction effects are shown and displacement evaluation gives similar results for large motion and large diameter particles. This comparison has to be extending now to other blocks for different diameter particles and concentration.

\section{Acknowledgments}

The authors gratefully acknowledge the support of the French National Research Agency (VIVE3D) and the partial support of the CPER 13.

\section{References}

1. P. Arroyo, K.D. Hinsch, Particle Image Velocimetry, Topics Appl. Physics 112 (2008).

2. C. H. Atkinson, J. Soria, 16th Australasian Fluid Mechanics Conference, Australia, (2007).

3. G. E. Elsinga, F. Scarano, B. Wienele, B. W. Van Oudheusden, Exp. in Fluids 41 (2006).

4. A. Fincham, 5th International Symposium on Particle Image Velocimetry PIV'03, 3101, Busan, Korea, (2003).

5. A. Germaneau, P. Doumalin, J.C. Dupré, Exp. Mech., 47, 4 (2007).

6. A. Germaneau, P. Doumalin, J.C. Dupré, Strain, 43, 3 (2007).

7. Kaczmarz S. Bull. Internat. Acad. Poln. Sci. Lettres A, (1937).

8. L. Thomas, V. Grulier, P. Braud, B. Tremblais, A. Germaneau, J.C. Dupré, P. Doumalin, L. David, 8th International Symposium on Particle Image Velocimetry, Melbourne, Victoria, Australia, August 25-28, (2009).

9. G. E. Elsinga, B. W. Van Oudheusden, F. Scarano, 13th Int. Symp. on Applications of Laser Techniques to Fluid Mech, 20.5, (2006). 